\title{
Recent Results on Searches for Heavy Majorana Neutrinos
}

\section{Jon Harrison}

on behalf of the LHCb, BABAR and Belle collaborations

The $13^{\text {th }}$ International Workshop on Tau Lepton Physics Aachen, Wednesday $17^{\text {th }}$ September, 2014
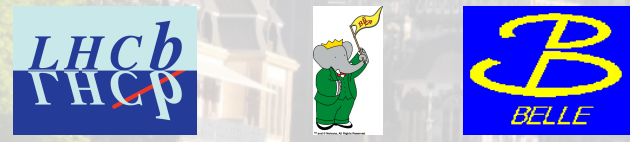

\section{MANCHESTER 1824}




\section{Heavy Majorana neutrinos at $\mathrm{LHCb}$ and the $B$ factories}

$\rightarrow$ Majorana neutrinos are theoretically well motivated

$\rightarrow$ See previous talks (e.g. F. Deppisch) for more information

$\rightarrow$ Focus today on searches for the decays of heavy mesons to final states with two same sign leptons
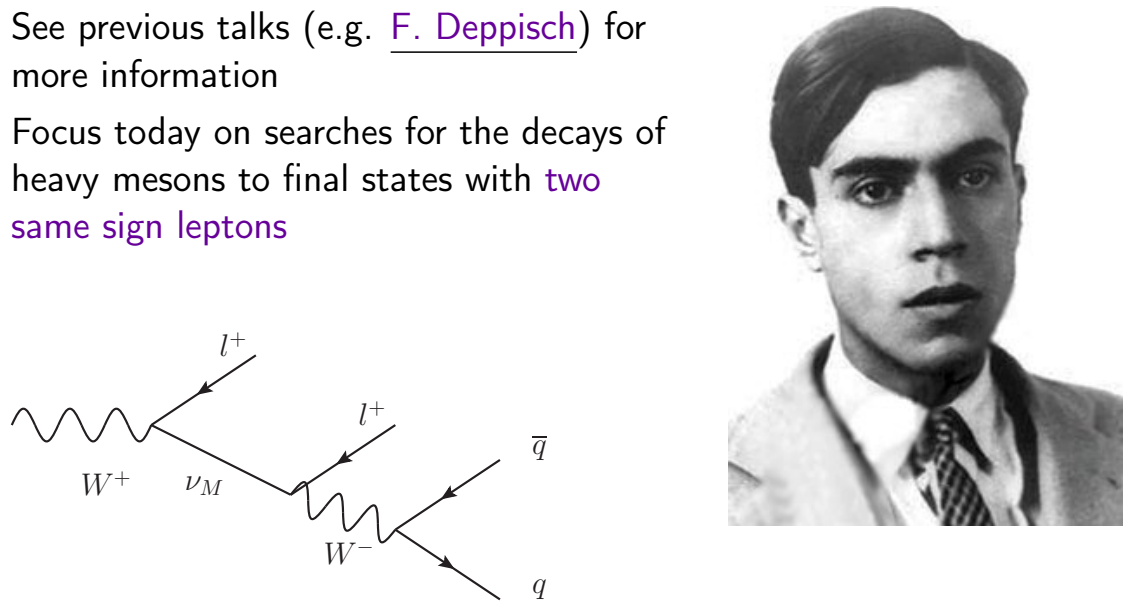


\section{The LHCb Detector}

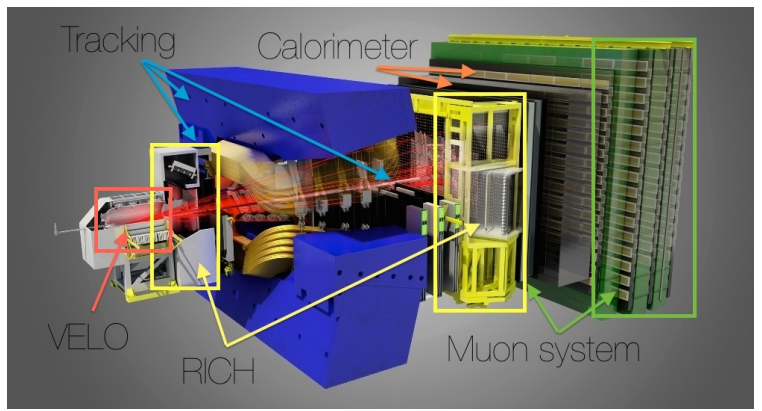

A dedicated flavour physics experiment in the forward region at the LHC

$3.0 \mathrm{fb}^{-1}$ of integrated luminosity from $p p$ collisions at 7 and $8 \mathrm{TeV}$

$\rightarrow$ Precise vertex reconstruction: $<10 \mu \mathrm{m}$ vertex resolution in $x$ and $y$

$\rightarrow$ Excellent charged particle separation: $\pi^{ \pm}$misID of $10 \%$ for $95 \% K^{ \pm}$ efficiency

$\rightarrow$ Clean identification of muons: misID of $1 \%$ for $98 \% \mu^{ \pm}$efficiency

$\rightarrow$ Excellent mass resolution: typically $7-20 \mathrm{MeV}$

$\rightarrow$ Flexible low- $p_{T}$ trigger 


\section{Searches for Majorana neutrinos in $B^{+} \rightarrow h^{-} \mu^{+} \mu^{+}$at} $\mathrm{LHCb}$

$\rightarrow B^{+} \rightarrow h^{-} \mu^{+} \mu^{+}$decays probe a wide range of Majorana neutrino masses

$\rightarrow$ Search performed for a range of neutrino lifetimes

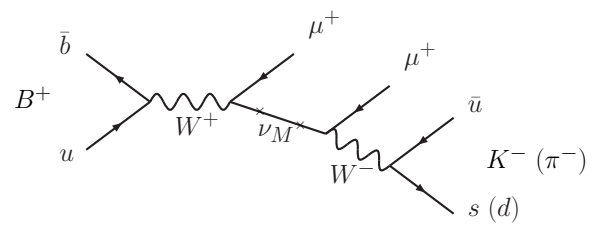

$\rightarrow$ Results from $3 \mathrm{LHCb}$ papers:

LHCb-PAPER-2013-064, LHCb-PAPER-2011-038 and LHCb-PAPER-2011-009

$\rightarrow$ Encompass $h^{-}=\pi^{-}, D^{-}, D^{*-}, D_{s}^{-}$and $D^{0} \pi^{-}$and $K^{-}$ 


\section{Analysis strategy}

$\rightarrow$ Results use between $36 \mathrm{pb}^{-1}$ (7 $\mathrm{TeV})$ and $3.0 \mathrm{fb}^{-1}(7 \mathrm{TeV}+8$ $\mathrm{TeV})$ of LHCb data

$\rightarrow$ Split into short $(\mathcal{S})$ and long $(\mathcal{L})$ neutrino lifetimes due to detached vertex

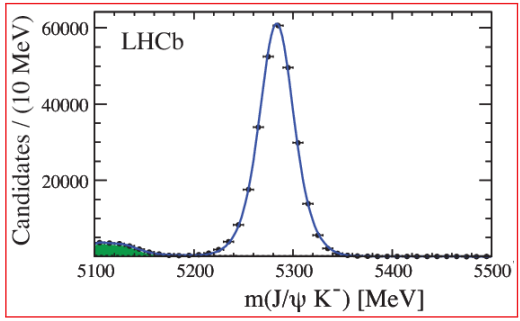

$\rightarrow$ Normalise to $\mathrm{B}^{+} \rightarrow \mathrm{J} / \psi \mathrm{K}^{+}$ (3-body) and $\mathrm{B}^{+} \rightarrow \psi(2 S) \mathrm{K}^{+}$ (5-body) with charmonium backgrounds (green) estimated from data

$\rightarrow$ Search for signal in $2 \sigma$ around $B^{+}$mass

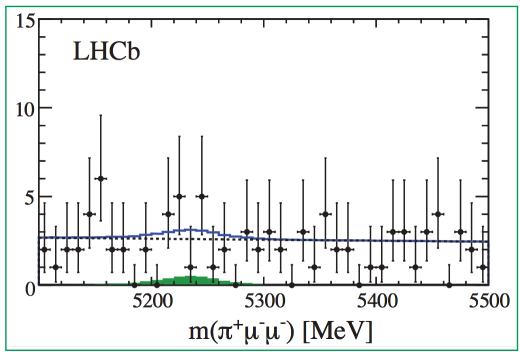

$\mathcal{S}$ data 


\section{Mass and lifetime dependence}

$\rightarrow$ No signal observed

$\rightarrow$ Limit for $\mathcal{S}$ from average detection efficiency using $\mathrm{CL}_{\mathrm{s}}$ method

$\rightarrow$ Also scan limit in $5 \mathrm{MeV}$ steps of neutrino mass from 250 $5000 \mathrm{MeV}$ with varying $\sigma$

$\rightarrow$ For $\mathcal{L}$ scan in both mass and neutrino lifetime

$\rightarrow$ Dominant systematic from $\mathcal{B}\left(B^{+} \rightarrow J / \psi K^{+}\right)$

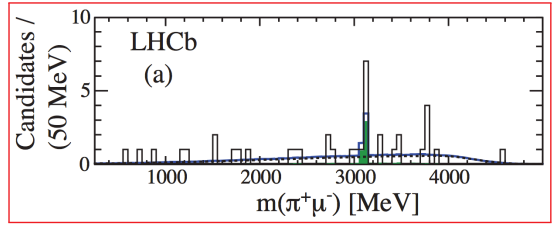

$\mathcal{S}$ data

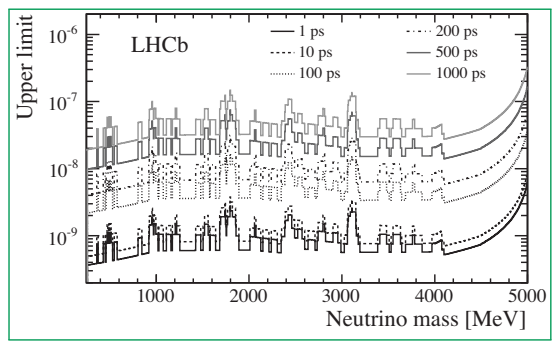




\section{$B^{+} \rightarrow h^{-} \mu^{+} \mu^{+}$results}

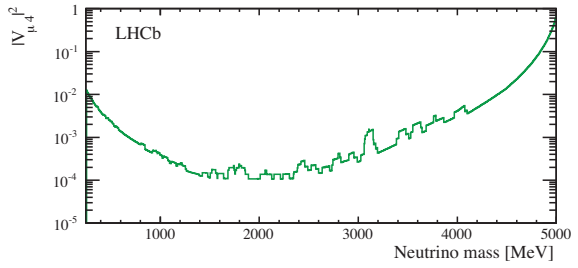

$$
B^{+} \rightarrow \pi^{-} \mu^{+} \mu^{+} \text {only }
$$

$\rightarrow$ All limits are world's bests

$\rightarrow$ improved by as much as

$\sim \times 100$ $\rightarrow$ Limits on fourth generation couplings, $\left|V_{\mu 4}\right|^{2}$, as a function of neutrino mass

\begin{tabular}{cc}
\hline$B^{+} \rightarrow K^{-} \mu^{+} \mu^{+}$ & $5.4 \times 10^{-8}$ \\
$B^{+} \rightarrow D^{-} \mu^{+} \mu^{+}$ & $6.9 \times 10^{-7}$ \\
$B^{+} \rightarrow D^{*-} \mu^{+} \mu^{+}$ & $2.4 \times 10^{-6}$ \\
$B^{+} \rightarrow \pi^{-} \mu^{+} \mu^{+}$ & $4.0 \times 10^{-9}$ \\
$B^{+} \rightarrow D_{s}^{-} \mu^{+} \mu^{+}$ & $5.8 \times 10^{-7}$ \\
$B^{+} \rightarrow D^{0} \pi^{-} \mu^{+} \mu^{+}$ & $1.5 \times 10^{-6}$ \\
\hline
\end{tabular}




\section{Search for Majorana neutrinos in $D_{(s)}^{+} \rightarrow \pi^{-} \mu^{+} \mu^{+}$at $\mathrm{LHCb}$}

$\rightarrow$ Majorana neutrino-mediated $D_{(s)}^{+} \rightarrow \pi^{-} \mu^{+} \mu^{+}$decays can occur in a similar manner to $B^{+} \rightarrow h^{-} \mu^{+} \mu^{+}$

$\rightarrow$ World's best experimental limits from BABAR of $2 \times 10^{-6}$ for $D^{+} \rightarrow \pi^{-} \mu^{+} \mu^{+}$and $1.4 \times 10^{-5}$ for $D_{s}^{+} \rightarrow \pi^{-} \mu^{+} \mu^{+}$

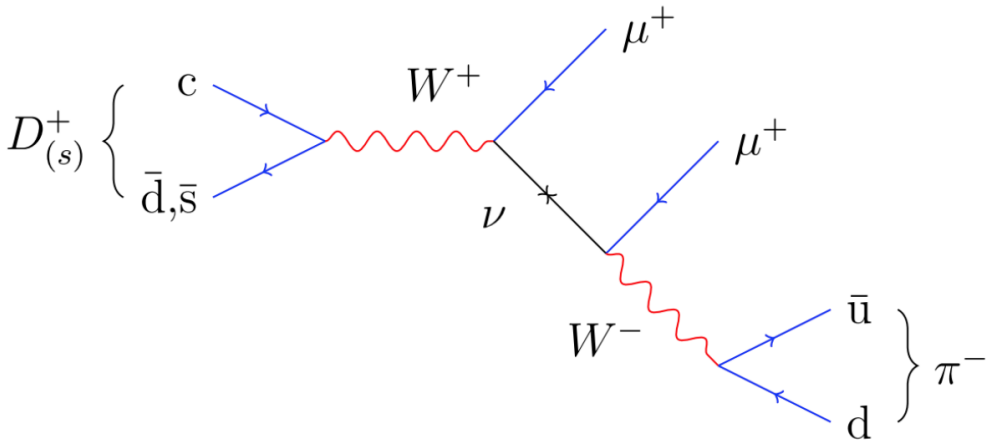




\section{$D_{(s)}^{+} \rightarrow \pi^{-} \mu^{+} \mu^{+}$results}

$\rightarrow$ Analysis uses $1.0 \mathrm{fb}^{-1}$ of $7 \mathrm{TeV}$ data collected in 2011

$\rightarrow$ Normalisation to $D_{(s)}^{+} \rightarrow \phi\left(\mu^{+} \mu^{-}\right) \pi^{+}$

$\rightarrow$ Classification of signal and background from PID cuts and a BDT using kinematic and geometric variables, trained on 2010 data

$\rightarrow$ Peaking background from $D_{(s)}^{+} \rightarrow \pi^{+} \pi^{+} \pi^{-}$decays with shape (dark fill) measured from data

$\rightarrow$ Fit in bins of $m\left(\pi^{-} \mu^{+}\right)$to improve statistical significance

$\rightarrow$ Limit of $2.2 \times 10^{-8}$ for $D^{+}$and $1.2 \times 10^{-7}$ for $D_{s}^{+}$decays $(90 \% \mathrm{CL})$ are a factor of fifty

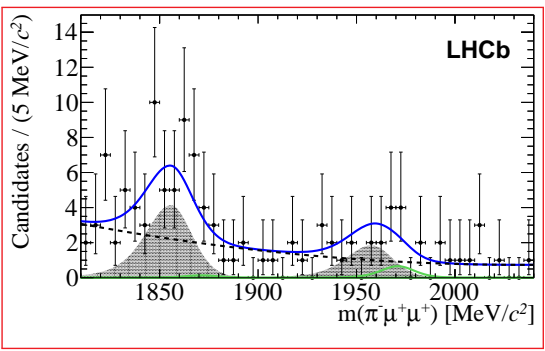

LHCb-PAPER-2012-051 improvement 


\section{The BABAR and Belle detectors}

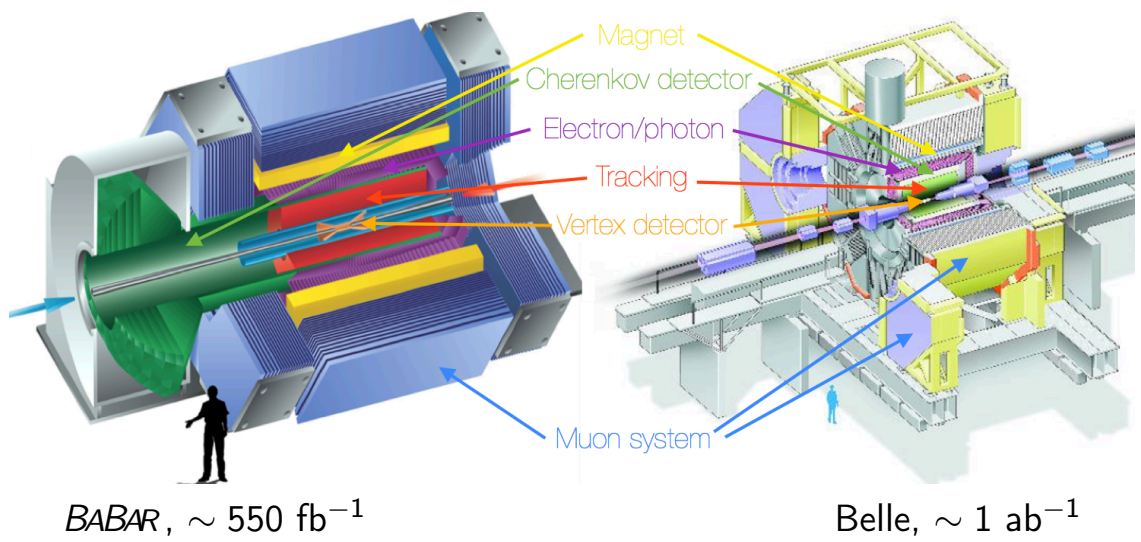

$\rightarrow$ Data collected mostly at the $\Upsilon(4 S)$ resonance

$\rightarrow$ Excellent $K / \pi$ separation; $\gamma$, e and $\mu$ identification 


\section{Search for $B^{+} \rightarrow X^{-} I^{+} I^{+}$at BABAR}

$\rightarrow$ Search for $11 \mathrm{LNV}$ processes $B^{+} \rightarrow X^{-} I^{+} I^{+}$with $X^{-}=K^{-}, \pi^{-}, \rho^{-}\left(\pi^{-} \pi^{0}\right), K^{*-}\left(K_{s}^{0} \pi^{-} / K^{-} \pi^{0}\right)$, or $D^{-}\left(K^{+} \pi^{-} \pi^{-}\right)$ and $I^{+} I^{+}=e^{+}$or $\mu^{+}$using $471 \pm 3$ million $B \bar{B}$ pairs

$\rightarrow$ Previously published (here) $h^{-}=K^{-}, \pi^{-}$with $I^{+} I^{+}=e^{+} e^{+}$or $\mu^{+} \mu^{+}$

$\rightarrow$ Use MC for selection efficiencies, systematics and backgrounds from $e^{+} e^{-} \rightarrow q \bar{q}$, Bhabha elastic scattering, Drell Yan, $B \bar{B}$ and diphoton events

$\rightarrow$ Require 4 charged tracks, with 2 or more leptons that pass PID and kinematic criteria

$\rightarrow$ Veto photon conversions and misidentified $J / \psi$ decays

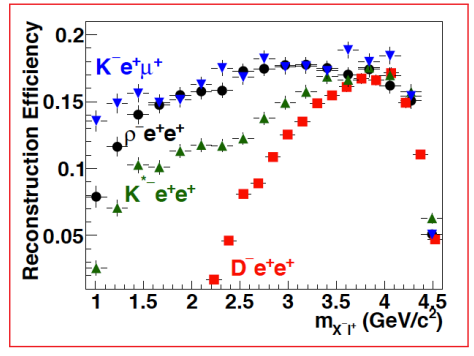

BABAR-PUB-13/016 


\section{Signal and background discrimination}

$\rightarrow$ Suppress backgrounds with cuts on:

1) $m_{E S}=\sqrt{\left(s / 2+\mathbf{p}_{0} \cdot \mathbf{p}_{\mathrm{B}}\right)^{2} / E_{0}^{2}-\mathbf{p}_{\mathrm{B}}{ }^{2}}$

2) $\Delta E=E_{B}^{*}-\sqrt{s} / 2$

3) BDT with 9 input variables trained using simulation and on-resonance data

$\rightarrow$ For multiple candidates select the one

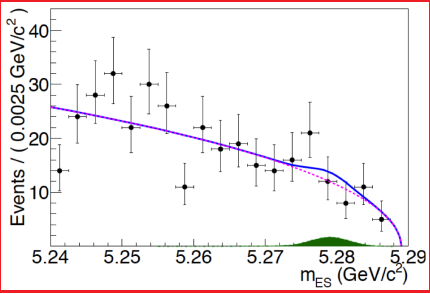
with the smallest $B$ vertex fit $\chi^{2}$

$\rightarrow$ Signal yields from unbinned maximum likelihood fit to $m_{E S}, \Delta E$, resonance mass (if applicable) and BDT output

$\rightarrow$ Test fit quality on toy MC, background samples and blinded on-resonance data

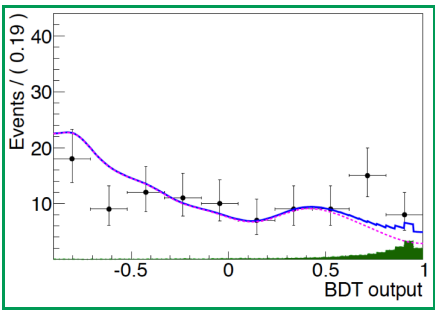




\section{BABAR results}

$\rightarrow$ Use a Bayesian approach to set 90\% CL upper limits

$\rightarrow$ Statistical uncertainties are dominant

$\rightarrow$ Limits in the range $(1.5-26) \times 10^{-7}$

$\rightarrow$ Limits on the $\rho^{-}, \pi^{-}$and $K^{-}$ modes are an order of magnitude improvement on previous results

\begin{tabular}{cc}
\hline \multicolumn{1}{c}{ Channel } & $\mathcal{B}_{U L}\left(\times 10^{-7}\right)$ \\
\hline$B^{+} \rightarrow K^{*-} e^{+} e^{+}$ & 4.0 \\
$B^{+} \rightarrow K^{*-} e^{+} \mu^{+}$ & 3.0 \\
$B^{+} \rightarrow K^{*-} \mu^{+} \mu^{+}$ & 5.9 \\
$B^{+} \rightarrow \rho^{-} e^{+} e^{+}$ & 1.7 \\
$B^{+} \rightarrow \rho^{-} e^{+} \mu^{+}$ & 4.7 \\
$B^{+} \rightarrow \rho^{-} \mu^{+} \mu^{+}$ & 4.2 \\
$B^{+} \rightarrow D^{-} e^{+} e^{+}$ & 26 \\
$B^{+} \rightarrow D^{-} e^{+} \mu^{+}$ & 21 \\
$B^{+} \rightarrow D^{-} \mu^{+} \mu^{+}$ & 17 \\
$B^{+} \rightarrow K^{-} e^{+} \mu^{+}$ & 1.6 \\
$B^{+} \rightarrow \pi^{-} e^{+} \mu^{+}$ & 1.5 \\
\hline
\end{tabular}




\section{Search for heavy neutrinos at Belle}

$\rightarrow$ Direct search for $\nu_{h} \rightarrow I^{ \pm} \pi^{\mp}$ using $B \rightarrow X I \nu_{h}$ with $X=D^{(*)}$, a light meson, or nothing and $I=e$ or $\mu$ using 772 million $B \bar{B}$ pairs

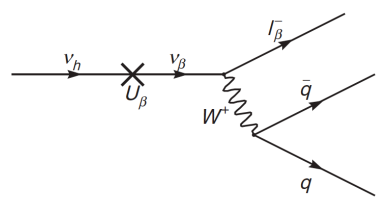

$\rightarrow \mathrm{MC}$ for generic background samples and signal efficiencies

$\rightarrow$ Partial reconstruction technique to increase sensitivity to large neutrino flight distances, require $>4$ charged tracks with PID and kinematic cuts

$\rightarrow$ Split analysis into small mass $\left(D^{(*)} / \nu_{h}\right)$ and large mass (all other)

$\rightarrow$ Efficiency depends on the neutrino mass and $B$ meson decay mode

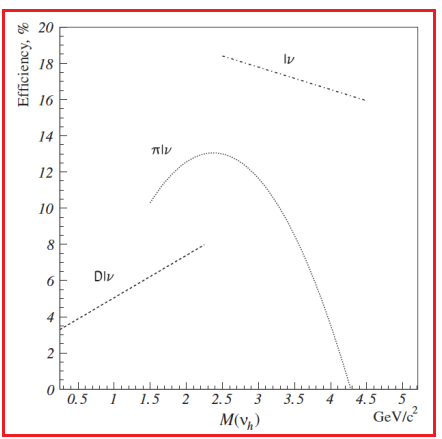

Belle 2012-28 


\section{Analysis details}

$\rightarrow$ In the small mass analysis reduce backgrounds via:

1) The recoil mass, $M_{X}=$

$\left(E_{C M}-E_{l / \pi}\right)^{2}-P_{\| / \pi}^{2}-P_{B}^{2}$, which peaks at the $D^{(*)}$ mass

2) A proton veto using PID

$\rightarrow$ Few remaining events are consistent with background expectations and are categorised depending on hits in the SVD

$\rightarrow$ Number of detected neutrinos is proportional to the coupling constants between Majorana and

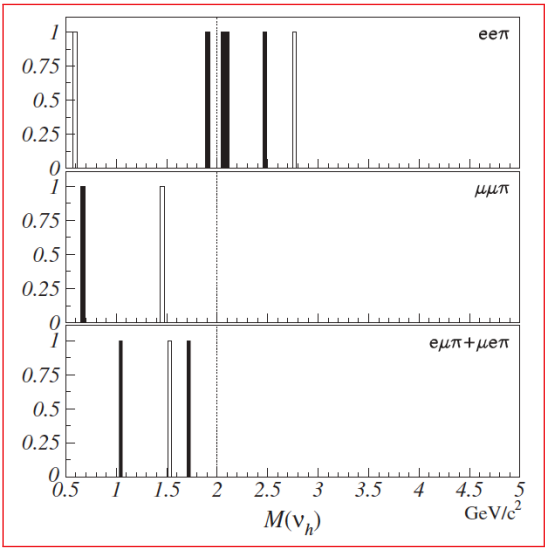
SM neutrinos 


\section{Belle results}

$\rightarrow$ Set upper limits on the coupling constants $\left|U_{e}\right|^{2},\left|U_{\mu}\right|^{2}$ and $\left|U_{e}\right|\left|U_{\mu}\right|$ from ee $\pi, \mu \mu \pi$ and $e \mu \pi+\mu e \pi$ decay modes using Feldman Cousins

$\rightarrow$ Maximum sensitivities around $2 \mathrm{GeV} / c^{2}$ of $3.0 \times 10^{-5}$, $3.0 \times 10^{-5}$ and $2.1 \times 10^{-5}$ for $\left|U_{e}\right|^{2},\left|U_{\mu}\right|^{2}$ and $\left|U_{e}\right|\left|U_{\mu}\right|$

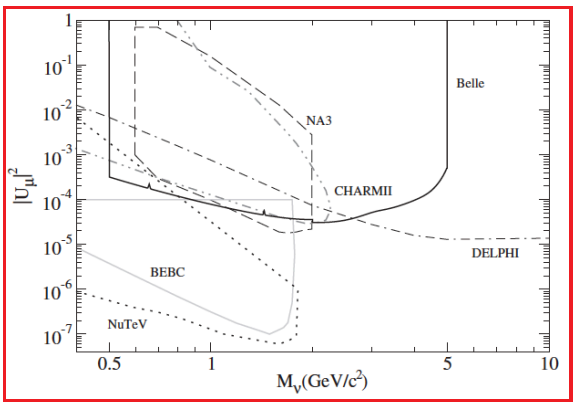

Corresponds to an upper limit (at $90 \% \mathrm{CL}$ ) of $\mathcal{B}\left(B \rightarrow X I \nu_{h}\right) \times \mathcal{B}\left(\nu_{h} \rightarrow I \pi^{+}\right)<7.2 \times 10^{-7}$ for $I=e$ or $\mu$ 


\section{Summary}

A number of new results on searches for Heavy Majorana neutrinos

$\rightarrow$ World's best limits on $B^{+} \rightarrow h^{-} \mu^{+} \mu^{+}$and $D_{(s)}^{+} \rightarrow \pi^{-} \mu^{+} \mu^{+}$decays from $\mathrm{LHCb}$

$\rightarrow$ The $B$ factories continue to exploit their datasets

$\rightarrow$ Expect further results from LHCb following the restart of the LHC

Even more to come in the future from Belle 2 and the LHCb Upgrade

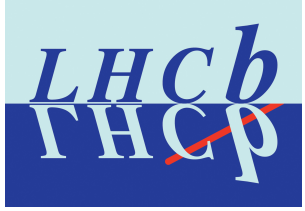

\section{MANCHESTER 1824}




\section{Backup}




\section{$\mathrm{LHCb}$ integrated luminosity}

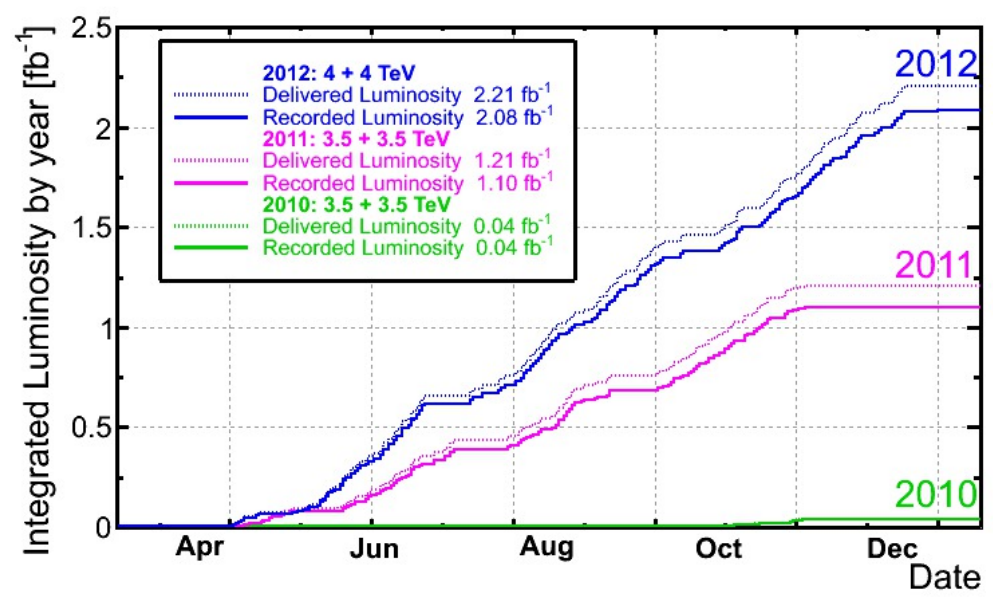




\section{The LHCb trigger: $2010+2011$}

$\rightarrow$ The trigger reduces the event rate via:

(1) L0: Hardware selection using calo clusters and muon system hits

(2) HLT1: Loose software selection using VELO and tracking station tracks

(3) HLT2: Full software reconstruction creates composite particles

$40 \mathrm{MHz}$ bunch crossing rate
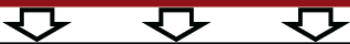

LO Hardware Trigger : $1 \mathrm{MHz}$ readout, high $E_{\mathrm{T}} / \mathrm{P}_{\mathrm{T}}$ signatures

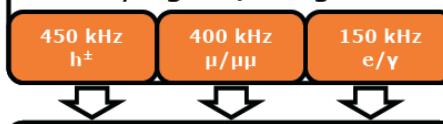

\section{Software High Level Trigger 29000 Logical CPU cores}

Offline reconstruction tuned to trigger time constraints

Mixture of exclusive and inclusive selection algorithms

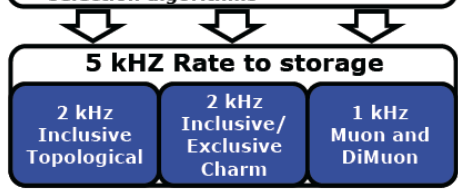




\section{The LHCb trigger: 2012}

$\rightarrow$ Introduction of partial deferred triggering in 2012

$\rightarrow 20 \%$ of events passing LO saved to disk and processed inter-fill

$\rightarrow$ Effective 20\% increase in CPU power

$\rightarrow$ Used to reduce track $p_{T}$ thresholds from 500 to $300 \mathrm{MeV}$

$\rightarrow$ significant trigger efficiency improvements for decays with low $p_{T}$ tracks

$\rightarrow$ Further improvements for Run II
$40 \mathrm{MHz}$ bunch crossing rate
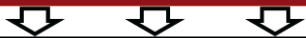

Lo Hardware Trigger : 1 MHz readout, high $E_{\mathrm{T}} / \mathrm{P}_{\mathrm{T}}$ signatures

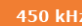

$\mathrm{h}^{ \pm}$

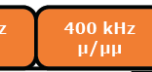

$150 \mathrm{kHz}$ e/v

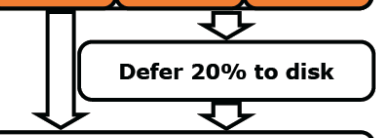

Software High Level Trigger 29000 Logical CPU cores

Offline reconstruction tuned to trigger time constraints

Mixture of exclusive and inclusive selection algorithms

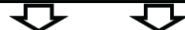

5 kHZ Rate to storage 